\title{
Síndrome Respiratória Aguda Grave em Pernambuco: comparativo dos padrões antes e durante a pandemia de COVID-19
}

\author{
Severe Acute Respiratory Syndrome in Pernambuco: \\ comparison of patterns before and during the COVID-19 pandemic
}

Amanda Priscila de Santana Cabral Silva (https://orcid.org/0000-0003-2337-9925) 1,2

Lívia Teixeira de Souza Maia (https://orcid.org/0000-0002-0161-7729) ${ }^{1}$

Wayner Vieira de Souza (https://orcid.org/0000-0002-0939-9332)2

${ }^{1}$ Núcleo de Saúde Coletiva, Centro Acadêmico Vitória, Universidade Federal de Pernambuco. R. Alto do Reservatório s/n, Bela Vista. 55608-680 Vitória de Santo Antão PE Brasil. amanda. cabral@ufpe.br

${ }^{2}$ Departamento de Saúde Coletiva, Instituto Aggeu Magalhães, Fundação Oswaldo Cruz. Recife PE Brasil.

\begin{abstract}
The aim of this study was to analyze the Severe Acute Respiratory Syndrome (SARS) pattern in Pernambuco before and during a COVID-19 pandemic. Ecological study conducted from January to June, 2015 to 2019 and from January 1 to June 15, 2020. The detection rates by municipality and by Regional Health of residence were calculated. The spatial area of SARS was estimated through the risk ratio. Before the pandemic, there were 5,617 cases of SARS, 187 cases/month and 23.8 cases/100 thousand inhabitants, while during the pandemic there were 15,100 cases, 2,516 cases/month and $320.3 \mathrm{ca}$ ses/100 thousand inhabitants, which represents a 13-fold increase in detection. The following expanded $(p<0,001)$ : the occurrence in elderly people, the collection of samples and the identification of SARS etiological agent with predominance of SARS by COVID-19. Most municipalities experienced a 20-fold higher detection than expected, suggesting a process of virus spread to the hinterlands. The excess risk associate with lower IDHM, the condition of the municipality being the headquarters of the Regional Health and the presence of a highway in the municipality. The change in the pattern of occurrence of SRAG, combined with Spatial analysis may contribute to action planning at different levels of management.
\end{abstract}

Key words COVID-19, Pandemics, Epidemiological monitoring, Spatial analysis
Resumo Foi analisado o padrão da Síndrome Respiratória Aguda Grave (SRAG) em Pernambuco antes e durante período pandêmico da COVID-19. Estudo ecológico, de janeiro a junho de 2015 a 2019 (período pré-pandemia) e 01 de janeiro a 15 de junho de 2020, período que contempla parte inicial da pandemia. Taxas de deteç̧ão por município e Regional de Saúde de residência foram calculadas e, por meio da razão de risco, a dinâmica espacial da SRAG foi estimada. Na pré-pandemia ocorreram 5.617 casos de SRAG, 187 casos/mês e 23,8 casos $/ 100$ mil hab. e, na pandemia, 15.100 casos, 2.516 casos/ mês e 320,3 casos/100 mil hab., um aumento da detecção mensal em 13 vezes. Foi ampliada ( $p$ $<0,001$ ) a ocorrência em idosos (de 7,7\% para $48,3 \%$ ), a coleta de amostras (de 60,9\% para 96,5\%) e a identificação do agente etiológico causador da SRAG, com predominio da SRAG por COVID-19 (66,0\%). Regiões do interior do estado tiveram razão de deteç̧ão de SRAG 20 vezes superior à esperada, sendo o excesso de risco associado a menor IDHM, a condição do município ser sede de Regional de Saúde e a presença de rodovia federal no território municipal. Conhecer a mudança do padrão da ocorrência da SRAG, aliada a análise espacial poderão contribuir para o planejamento de ações a curto e médio prazo nos diferentes níveis de gestão.

Palavras-chave COVID-19, Pandemia, Vigilância epidemiológica, Análise espacial 


\section{Introdução}

A vigilância da Síndrome Respiratória Aguda Grave (SRAG) foi criada em 2009, em decorrência da pandemia de influenza pelo vírus H1N1. O sistema, inicialmente limitado a notificar acometidos pelos vírus influenza no país, se aperfeiçoou e incorporou o acompanhamento de outros vírus respiratórios, aumentando a abrangência da vigilância ${ }^{1}$.

Desde setembro de 2012 o caso de SRAG compulsoriamente notificado ao Sistema Nacional de Agravos de Notificação (Sinan) é definido como o indivíduo de qualquer idade, hospitalizado com síndrome gripal (febre e tosse ou dor de garganta) e que apresente dispneia ou saturação de $\mathrm{O}_{2}$ menor que $95 \%$ ou desconforto respiratório. Também devem ser registrados os óbitos por SRAG independente da internação $0^{1,2}$.

No Brasil, até a semana epidemiológica 52 de 2019 foram notificados 40.294 casos de SRAG, sendo $14,2 \%$ classificados como SRAG por influenza, $19,3 \%$ causada por outros vírus respiratórios, $0,4 \%$ por outros agentes etiológicos, $59,7 \%$ não especificados e $6,4 \%$ ainda em investigação. O Nordeste respondeu por $16,2 \%$ dos casos de SRAG do país $(\mathrm{n}=6.612)$, sendo Pernambuco o maior notificador da região, com aproximadamente $40 \%$ dos casos $(n=2.486)$. No estado, $5,2 \%$ das notificações foram confirmados para Influenza; outros agentes etiológicos e outros vírus respiratórios responderam por $0,1 \%$ do total de casos; $76,8 \%$ não foi especificada e $17,9 \%$ estão em investigação ${ }^{3}$.

O caráter sindrômico dessa vigilância permite monitorar o comportamento dos vírus respiratórios sazonais e a introdução de novos agentes etiológicos. Em dezembro de 2019, um surto de uma nova doença de coronavírus (COVID-19, causada pelo Coronavírus 2 da Síndrome Respiratória Aguda Grave -SARS-CoV-2) foi relatado em Wuhan, China, se apresentando como um dos maiores desafios sanitários em escala global deste século ${ }^{4}$. Na metade do mês de abril, poucos meses depois do início da epidemia, já havia ocorrido mais de 2 milhões de casos e 120 mil mortes no mundo por COVID-194. No Brasil, no mesmo período, foram registrados cerca de 28 mil casos confirmados e 1.745 mortes pela doença ${ }^{5}$.

A apresentação clínica se assemelha a sintomas leves de pneumonia viral e a gravidade da doença varia de leve a grave. Aproximadamente $80 \%$ dos pacientes apresentam doença leve, $15 \%$ apresentam doença grave e 5\% apresentam doença crítica ${ }^{6}$. Relatórios iniciais sugerem que a gravidade da doença está associada à idade avançada e à presença de comorbidades ${ }^{6,7}$. Além de requerer mudanças radicais de comportamento, nos níveis individual e comunitário, a COVID-19 tem exigido respostas rápidas no que se refere a ações preventivas, ampliação e a aquisição de insumos e leitos hospitalares, redimensionamento de recursos humanos em saúde, dentre outras estratégias de controle ${ }^{8,9}$.

Futuramente é esperado que o SARS-CoV-2 esteja entre os vírus respiratórios sazonais no país, permanecendo na rotina de monitoramento da vigilância universal de SRAG. Entretanto a possível existência de diferenças de ocorrência da SRAG num cenário pré-pandemia e durante a pandemia da COVID-19, pode influenciar a rotina desta vigilância no pós-pandemia. Torna-se relevante uma investigação para contribuir com ações de planejamento que promovam a equidade na atenção à saúde e na vigilância epidemiológica, com destaque para os níveis municipais e regionais.

Desta forma, este estudo teve como objetivo analisar o padrão da SRAG em Pernambuco, antes e durante a pandemia de COVID-19.

\section{Métodos}

Trata-se de um estudo ecológico que teve como local de estudo o estado de Pernambuco, situado na Região Nordeste do Brasil. Administrativamente, é dividido em 185 municípios distribuídos em 12 Regionais de Saúde, agrupadas em 4 Macrorregionais de Saúde ${ }^{10}$, sendo estas: Metropolitana (4 Regionais e 72 municípios); Agreste (2 Regionais e 53 municípios); Sertão (3 Regionais e 35 municípios e Vale do São Francisco e Araripe (3 Regionais e 25 municípios) (Figura 1). A população estimada para o ano de 2019 foi de 9.557.071 habitantes ${ }^{11}$.

Considerando a sazonalidade dos principais agentes etiológicos causadores de SRAG, foram considerados casos da doença os residentes em Pernambuco, detectados no período pré-pandemia (01 de janeiro a 30 de junho dos anos de 2015 a 2019) e no período que inclui o início da pandemia (01 de janeiro a 15 de junho de 2020). Nos anos de 2015 a 2018 as notificações foram realizadas no banco de dados do Sistema Nacional de Agravos de Notificação - Sinan Influenza Web e, a partir de 2019, os registros de SRAG foram no âmbito da plataforma Sivep-Gripe.

No plano de análise foram calculadas frequências absolutas, relativas e taxas de detecção mé- 
dia (por 100 mil habitantes) dos casos de SRAG por períodos do estudo (pré-pandemia e pandemia) para o estado de Pernambuco. Foram comparadas a distribuição dos casos de acordo com as variáveis faixa etária $(<10,10-39,40-59,60$ anos e mais), sexo (masculino e feminino), realização de coleta de amostra ( $\operatorname{sim}$, não) e classificação final (SRAG por influenza e por outros vírus respiratórios, SRAG por outro agente etiológico, SRAG por COVID-19, SRAG não especificada). Foi adotado o teste qui-quadrado de Pearson para identificar a existência de diferença com significância estatística.

Apesar da fonte de dados disponibilizar outras variáveis, foram consideradas aquelas onde a completude dos campos foi superior a $80 \%$. A análise consistiu apenas nos dados válidos. A exceção se deu para a variável classificação final, que embora tenha uma completude inferior a estipulada, traz informação relevante sobre o perfil dos agentes etiológicos da SRAG no período estudado.

Para a análise espacial, foram calculados para cada Regional de Saúde, para os dois períodos, o coeficiente de detecção de SRAG ([número de casos residentes /população residente] $\times 100.000$ habitantes).

Para a análise por município, considerando que pequenas populações e/ou subnotificações podem provocar variabilidade das taxas brutas, foi utilizado alisamento bayesiano empírico local, que permitiu suavizar as estimativas dos coeficientes calculados para pequenas (ou com subnotificação) áreas geográficas, eliminando flutuações aleatórias não associadas ao risco ${ }^{12}$.

A dinâmica espacial da detecção de SRAG no território de Pernambuco durante a pandemia foi apresentada por meio do cálculo da razão de risco (RR) entre as taxas de detecção, pré e durante a pandemia, a partir das taxas brutas para as Regionais de Saúde e das taxas suavizadas para os municípios.

Por fim foi investigada a associação entre a ocorrência das maiores razões de risco (o também chamado excesso de risco) entre as taxas de detecção municipais com indicadores socioeconômicos, sendo esses o Índice de Desenvolvimento Humano Municipal (IDHM) (Média geométrica dos índices das dimensões renda, educação e longevidade, com pesos iguais); Proporção de vulneráveis a pobreza (Proporção dos indivíduos com renda domiciliar per capita igual ou inferior a $1 / 2$ de salário mínimo em agosto de 2010); Proporção de extremamente pobres (Proporção dos indivíduos com renda domiciliar per capita igual ou inferior $1 / 4$ de salário mínimo em agosto de 2010); Presença de rodovia federal no território municipal; Município ser sede de

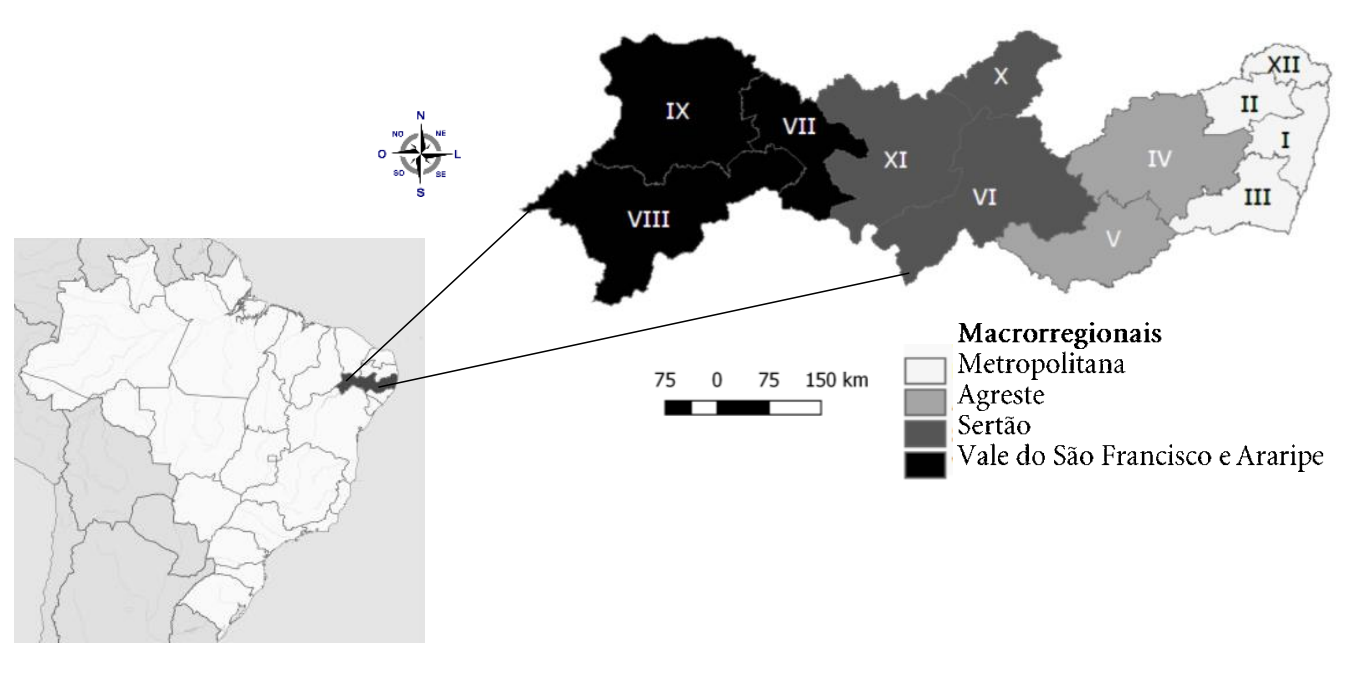

Figura 1. Macrorregionais e Regionais de Saúde. Pernambuco, Brasil, 2020. 
Regional de Saúde (coincidente com as sedes de polos de desenvolvimento econômico do estado).

Para identificação dos fatores associados, a razão de risco municipal (variável dependente) foi classificada em 0 (quando menor que a mediana) e 1 (quando igual ou maior que a mediana), permitindo calcular a regressão logística binária, do tipo backward Wald, adotando-se valor de $\mathrm{p}$ superior a 0,10 como critério para exclusão das variáveis do modelo.

Os softwares utilizados foram planilhas eletrônicas, SPSS V.22, TerraView V.4.2.2 e QGis 2.18.9. Os resultados foram representados na base cartográfica digital do estado de Pernambuco disponibilizada pelo Instituto Brasileiro de Geografia e Estatística (IBGE) ${ }^{13}$.

Os dados analisados neste estudo são de livre acesso, sendo os casos de SRAG disponibilizados na Plataforma Integrada de Vigilância em Saúde do site do Ministério da Saúde ${ }^{5}$; estimativas populacionais foram extraídas do IBGE ${ }^{11}$ e dados socio econômicos foram obtidos no atlas do desenvolvimento humano do Brasil ${ }^{14}$. O estudo utilizou base de dados secundários, publicamente disponíveis, sem possibilidade de identificação de indivíduos, sendo desnecessária submissão e aprovação em comitê de ética. Os autores garantem a confidencialidade e anonimato de todos os dados.

\section{Resultados}

No período pré-pandemia, entre 01 de janeiro e 30 de junho dos anos de 2015 a 2019, foram notificados 5.617 casos de SRAG residentes de Pernambuco, uma média de 187 casos por mês e taxa média semestral de detecção de 23,8 casos/100 mil hab. Entre 01 de janeiro a 15 de junho de 2020, período que inclui o início da pandemia, foram notificados 15.100 casos de SRAG, configurando uma detecção de 320,3 casos/100 mil habitantes e uma média de 2.517 de casos de SRAG/mês, o que representa um aumento de notificações em torno de 13 vezes no período recente. Com exceção do sexo, sendo o masculino predominante nos dois contextos, há diferenças entre o padrão da ocorrência de SRAG nos períodos analisados (Tabela 1). No período pré-pandemia, menores de 10 anos respondiam por $78,6 \%$ dos casos, enquanto na fase pandêmica $76,8 \%$ se concentraram entre os maiores de 40 anos, sendo $48,3 \%$ dos casos com 60 anos ou mais $\left(X^{2}=10670 ; \mathrm{p}<0,001\right)$.

A coleta de amostra clínica foi realizada em $60,9 \%$ dos casos no período pré-pandemia, sen- do ampliada para $96,5 \%$ dos casos durante a epidemia $\left(X^{2}=70,6 ; \mathrm{p}<0,001\right)$. No período anterior, $88,2 \%$ dos casos foram classificados como SRAG não especificada, seguido de $11,5 \%$ de casos com SRAG por Influenza e outros vírus respiratórios. Durante a pandemia $66,0 \%$ dos casos de SRAG foram por COVID-19, seguido de 2,5\% de casos com SRAG por Influenza e outros vírus respiratórios e em 31,2\% a SRAG não foi especificada (Tabela 1).

Até 2019 as Regionais de Saúde mais acometidas pela SRAG, com detecção superior a 20 casos/100 mil hab., se concentraram nas Macrorregionais Metropolitana e Sertão (Figura 2A). Durante o período estudado que contempla a pandemia, todas as Regionais de Saúde tiveram detecção superior a 60 casos/100 mil habitantes. Três das quatro regionais localizadas na Macrorregião Metropolitana apresentaram detecção superior a 200 casos/100 mil habitantes, chegando ao patamar de 575,0 casos/100 mil hab. na $1^{\text {a }}$ Regional de Saúde (Figura 2B).

Em todo o estado foi notado o excesso de risco de ocorrência de SRAG. Em todas as macrorregionais foram detectadas Regionais de Saúde com risco superior a 10 vezes a média esperada; destaca-se Regionais de Saúde localizadas nas Macrorregionais Sertão e Vale do São Francisco e Araripe que atingiram excesso de risco superior a 20 vezes da média esperada (Figura 2C).

No período pré-pandemia, $83,2 \%$ dos municípios $(\mathrm{n}=154)$ apresentaram taxa inferior à média de detecção anual (20 casos por $100 \mathrm{mil}$ habitantes) (Figura 3A). Apenas dois municípios tiveram mais de 60 casos/100 mil habitantes: Goiana (67,0 casos/100 mil hab.) e Afogados da Ingazeira (66,1 casos/100 mil hab.) (Figura 3A).

Mudança no padrão foi observada durante a pandemia, quando $98,4 \%$ dos municípios ( $\mathrm{n}=$ 182) tiveram taxa de detecção superior a 20 casos/100 mil habitantes (Figura 3B). Mais de 60 casos/100 mil habitantes foram contabilizados em $80 \%$ dos municípios $(\mathrm{n}=148)$ (Figura 3B). O excesso de risco revela que todos os municípios sofreram aumento da detecção de SRAG durante a pandemia. Contudo regiões mais afastadas da Região Metropolitana tiveram um maior impacto nesse aumento, como Vale do São Francisco e Araripe. Dos 25 municípios que formam a Macrorregião, 17 tiveram detecção superior a 10 vezes a esperada e em 12 destes a detecção foi 20 vezes maior a estimada (Figura 3C).

A análise multivariável, por meio da regressão logística binária, identificou três fatores associados ao excesso de risco de deteç̧ão de SRAG, 
Tabela 1. Comparativo do perfil da Síndrome Respiratória Aguda Grave segundo período pré-pandemia e durante pandemia de Covid-19. Pernambuco, 2015 a 2020.

\begin{tabular}{|c|c|c|c|c|c|}
\hline \multirow{2}{*}{ Variáveis analisadas } & \multicolumn{2}{|c|}{ Pré-pandemia ${ }^{*}$} & \multicolumn{2}{|c|}{ Pandemia ${ }^{\star *}$} & \multirow{2}{*}{$\mathrm{X}^{2}$; Valor de $\mathrm{p}$} \\
\hline & n & $\%$ & n & $\%$ & \\
\hline \multicolumn{6}{|l|}{ Faixa etária } \\
\hline$<10$ & 4417 & 78,6 & 1146 & 7,6 & $10670 ; \mathrm{p}<0,001$ \\
\hline $10-39$ & 489 & 8,7 & 2357 & 15,6 & \\
\hline $40-59$ & 277 & 4,9 & 4297 & 28,5 & \\
\hline$\geq 60$ & 434 & 7,7 & 7300 & 48,3 & \\
\hline \multicolumn{6}{|l|}{ Sexo } \\
\hline Masculino & 2989 & 53,2 & 8011 & 53,1 & 0,$014 ; \mathrm{p}=0,906$ \\
\hline Feminino & 2627 & 46,8 & 7067 & 46,9 & \\
\hline \multicolumn{6}{|l|}{ Realização de coleta de amostra } \\
\hline $\operatorname{Sim}$ & 3364 & 60,9 & 11649 & 96,5 & 70,$6 ; \mathrm{p}<0,001$ \\
\hline Não & 2160 & 39,1 & 417 & 3,5 & \\
\hline \multicolumn{6}{|l|}{ Classificação final } \\
\hline SRAG por influenza e outros vírus respiratórios & 628 & 11,5 & 196 & 2,5 & $5960 ; \mathrm{p}<0,001$ \\
\hline SRAG por outro agente etiológico & 18 & 0,3 & 28 & 0,4 & \\
\hline SRAG por COVID & 0 & 0,0 & 5235 & 66 & \\
\hline SRAG não especificada & 4807 & 88,2 & 2470 & 31,2 & \\
\hline
\end{tabular}

${ }^{\star} 01$ de janeiro a 30 de junho dos anos de 2015 a 2019, ${ }^{* *} 01$ de janeiro a 15 de junho de 2020.

Fonte: Elaboração própria a partir do Sinan Web Influenza e Sivep Gripe.

o IDHM, o município ser sede de Regional de Saúde e apresentar rodovia federal em seu território. O modelo inicial e final de regressão são apresentados na Tabela 2.

\section{Discussão e conclusões}

Este estudo comparou a ocorrência da SRAG em Pernambuco antes e durante ascensão da pandemia de COVID-19. Identificar um volume de notificações 13 vezes maior durante a pandemia, comparado com o período anterior sugere que mesmo passível a subnotificações, o sistema de vigilância da SRAG em Pernambuco é sensível a alterações da ocorrência da doença, tendo o potencial para captar epidemias e mudanças no perfil dos $\operatorname{casos}^{15}$.

Quanto a faixa etária, a maior concentração de casos passou, dos menores de 10 anos, para aqueles com 60 anos ou mais, compatível com situação encontrada no Brasil e no mundo $0^{2,16-18}$. A proteção aos idosos é estratégia prioritária, tendo em vista que eles constituem grupo com maior risco de complicações e de morte pela COVID-19 $9^{7,18}$

Em 15 de junho de 2020, Pernambuco apresentava 46.427 casos confirmados de COVID-19, incluindo casos leves e casos graves, com 4.994 casos internados e 3.959 pessoas que evoluíram para o óbito ${ }^{19}$. No mesmo período, a coleta de amostras para investigação laboratorial dos 15.100 casos de SRAG ocorridos no estado foi superior a 95\%, com a identificação de 5.235 casos de SRAG por COVID-19.

Frente a pandemia, a Secretaria Estadual de Saúde de Pernambuco priorizou a testagem laboratorial de casos graves, seguido de outros grupos populacionais, a exemplo de profissionais de saúde e da segurança pública, além dos óbitos suspeitos por COVID-1920. Embora a estratégia influencie na melhoria da qualificação do diagnóstico de SRAG, uma das principais discussões durante a pandemia é a importância da ampla testagem laboratorial na população. Crianças e aqueles que desenvolvem infecções assintomáticas podem contribuir para a propagação da doença e para o contágio em grupos mais propensos a complicações. Contudo, é fato que o país tem capacidade limitada de produção e realização de testes diagnósticos?

O Brasil figura entre os países com menor número de testes por habitante. Até o final do mês de maio de 2020, o país registrou 2,28 testes a cada 1.000 hab., a menor taxa entre os países da América do Sul, como Chile (28,59), Paraguai $(3,97)$, Argentina $(3,33)$ e Uruguai $(11,94)^{21}$. Nesse mesmo período a taxa de testagem de Pernam- 


\section{A}

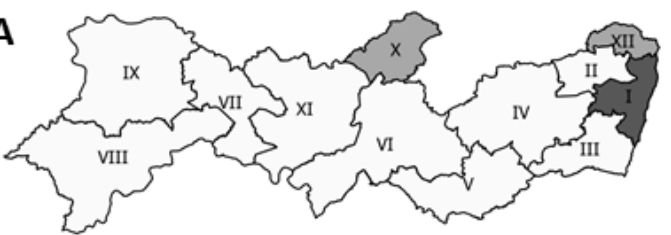

B
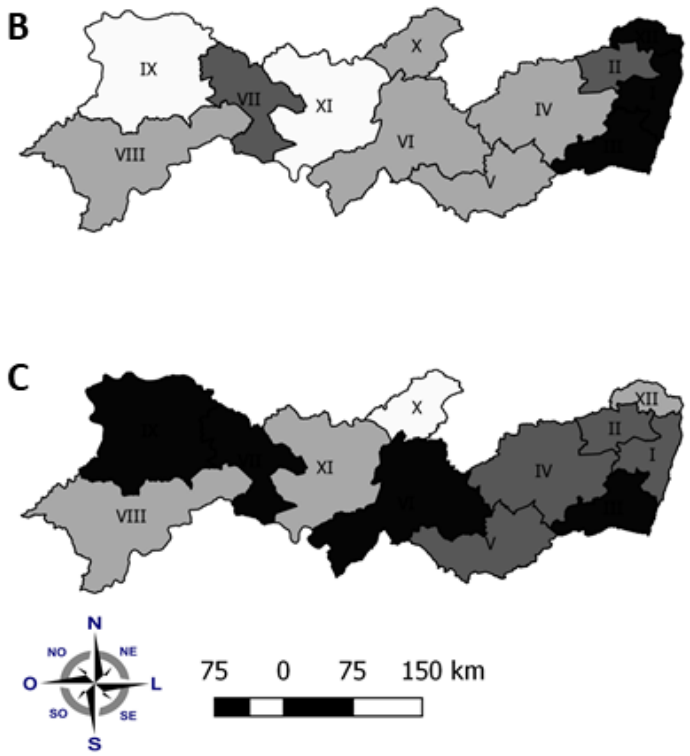

Legenda

Taxa de detecção Pré-Pandemia

$<=20,0(\mathrm{n}=9)$

$20,1-40,0(\mathrm{n}=2)$

$40,1-60,0(n=1)$

Legenda

Taxa de detecção Pandemia

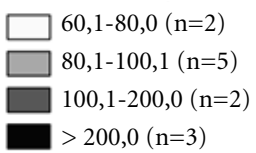

Legenda

Razão Risco

$\square<5,0(\mathrm{n}=1)$

$5,0-10,0(n=3)$

$10,1-20,0(n=4)$

$>20,0(n=4)$

Figura 2. Taxa de detecção de Síndrome Respiratória Aguda Grave (casos/100 mil habitantes) segundo Regional de Saúde de Residência. Pernambuco, Brasil. (A) Pré-pandemia (B) Pandemia (C) Razão de Risco.

Fonte: Elaboração própria.

buco era de 5,54 por 1.000 hab., mais do que o dobro da observada no Brasil ${ }^{22}$.

Quanto a classificação laboratorial final, a SRAG não especificada se destaca durante todo o período analisado, achado semelhante ao encontrado em estudo que analisou esse comportamento em todo o território nacional ${ }^{2}$. Além de questões relacionadas a qualidade da amostra coletada, manuseio inapropriado ou demora de processamento, deve ser considerada a circulação de outros agentes etiológicos diferentes aos rotineiramente testados ${ }^{2,23,24}$.

Embora o sistema tenha se aperfeiçoado, com a modificação do painel viral nos últimos anos, sabe-se que o vírus influenza sempre foi a prioridade desta vigilância ${ }^{1,2}$. A dinâmica populacional brasileira e os deslocamentos nacionais e interna- cionais, aliado a circulação de outros vírus respiratórios em outros países ${ }^{25,26}$ devem ser considerados no processo de ampliação do painel viral, o que pode influenciar na capacidade de identificação de novos agentes etiológicos e impactar no volume de amostras não especificadas.

A $1^{\text {a }}$ Regional de Saúde, que engloba a Região Metropolitana do Recife, apresentou a maior concentração de casos nos dois períodos. Esse território, apesar de responder por $44,3 \%$ da população do estado ${ }^{11}$, concentrou mais de $75 \%$ do total da SRAG. Esse espaço geográfico conta com uma alta densidade de estabelecimentos de saúde de alta complexidade de Pernambuco, e o acesso dessa população a essa rede mais sensível pode justificar tais achados no período pré-epidemia. Durante a epidemia, sobretudo no início, 
A

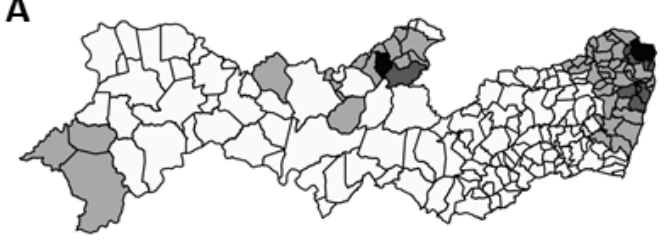

B

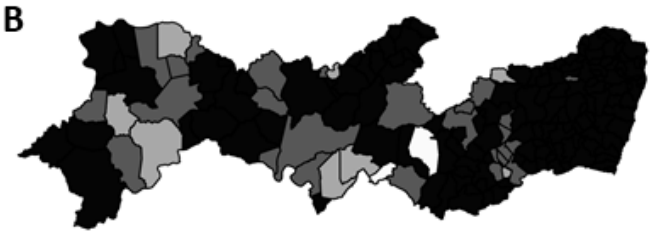

C

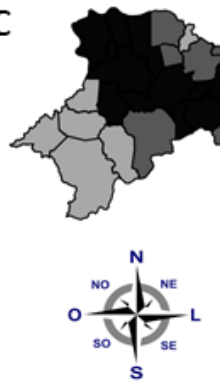

Legenda

Taxa de detecção Pré-Pandemia

$<=20,0(n=154)$

$20,1-40,0(n=19)$

$40,1-60,0(n=9)$

$>60,0(n=2)$
Legenda

Taxa de detecção Pandemia

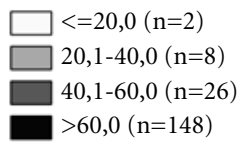

Legenda

Razão Risco

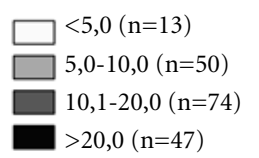

Figura 3. Taxa de detecção de Síndrome Respiratória Aguda Grave (casos/100 mil habitantes suavizada pelo estimador bayesiano local) segundo município de residência. Pernambuco, Brasil.

(A) Pré-pandemia (B) Pandemia (C) Razão de Risco.

Fonte: Elaboração própria.

um maior volume de notificações nessa região também pode estar relacionado ao tráfego aéreo e marítimo internacional, a alta densidade demográfica e mobilidade urbana, fatores que conhecidamente facilitam a circulação de inúmeros agentes etiológicos ${ }^{1,27,28}$.

A análise espacial, por meio da taxa de detecção suavizada, revela o espalhamento da doença em todo o estado de Pernambuco. A dinâmica de crescimento, entretanto, foi heterogênea, considerando as elevadas razões de risco encontradas no Agreste e no Vale do São Francisco e Araripe. Somado a esses achados está a associação encontrada entre o excesso de risco de detecção de SRAG a condição do município em ser sede de Regional de Saúde, ter rodovia federal em seu território ou ter IDHM reduzido.

A sede de Regional de Saúde é aqui um proxy de áreas desenvolvidas do ponto de vista econô- mico no estado. A associação encontrada, juntamente com a presença de rodovia federal no território municipal, sugere que o avanço da doença pode ser influenciado pelas relações sociais e econômicas que municípios do interior pernambucano guardam com o Recife, sendo essa uma característica comum entre cidades do interior nordestino e suas respectivas capitais ${ }^{27,29,30}$. Contudo, preocupa a também associação com baixos níveis de IDHM.

Conforme referido por Maciel et al. ${ }^{27}$, além da vulnerabilidade da população, baixos níveis de IDHM apontam para dificuldades nos serviços de saúde quanto ao diagnóstico e tratamento da doença. A disseminação de COVID-19 deverá afetar fortemente a infraestrutura no interior. Além disso, diferentemente de outras enfermidades em período não pandêmico, talvez não haja tempo hábil de transferência para as capitais ou 
Tabela 2. Modelo inicial e final da regressão logística para excesso de risco de detecção de Síndrome Respiratória Aguda Grave durante período pandêmico. Pernambuco, 2020*.

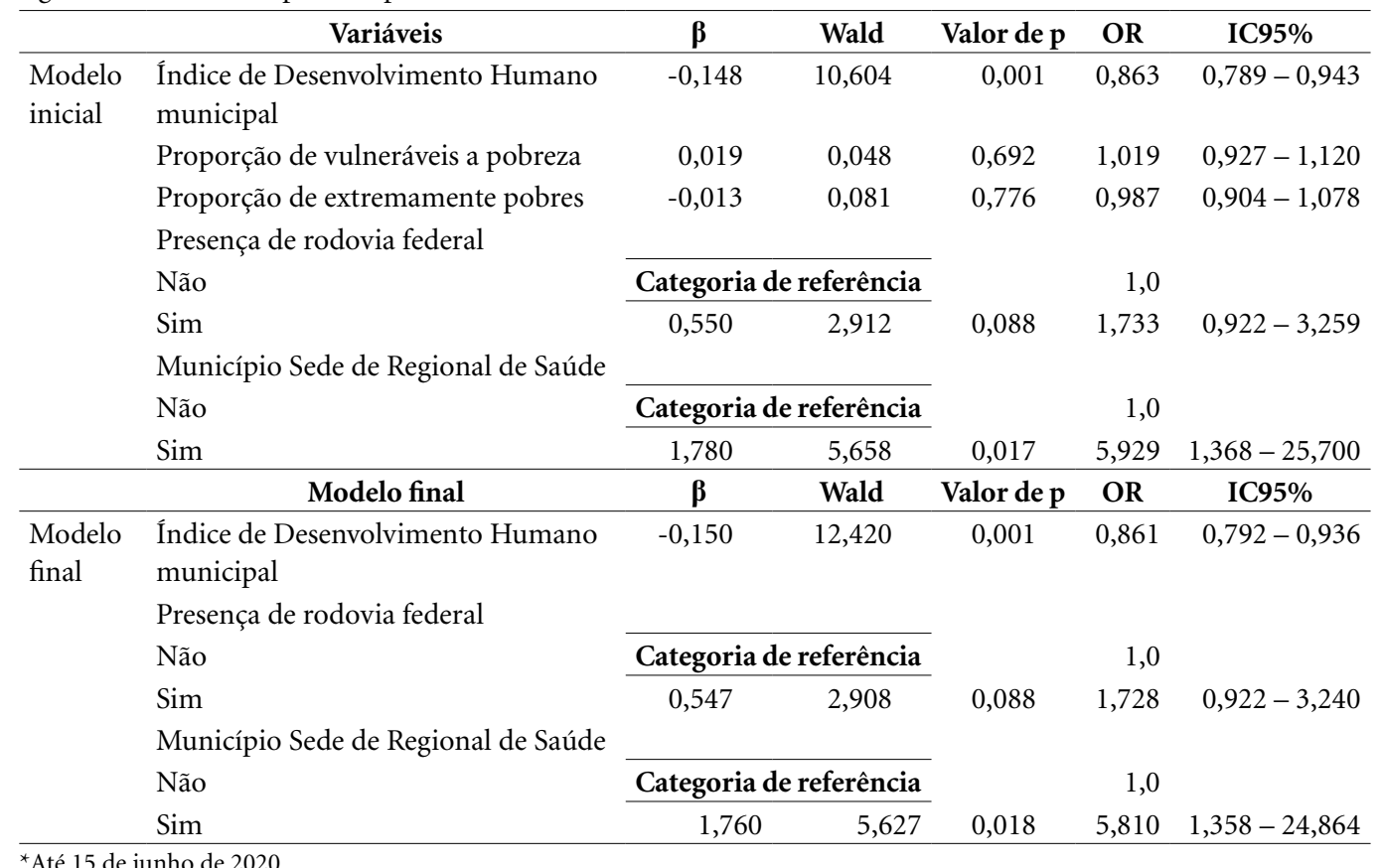

capacidade de atendimento para toda a demanda de pacientes graves, o que poderá refletir em diferentes coeficientes de letalidade associados às desigualdades sociais ${ }^{29,31}$.

Dentre as principais limitações do estudo se destacam a possível subnotificação de SRAG e a não utilização de outras variáveis, devido à baixa completude, para caracterizar o perfil da doença em Pernambuco. A complexidade da rotina de gestão de banco de dados em saúde, a exemplo da retirada de duplicidades e encerramento de casos, a dificuldades que levam ao atraso de processamento de amostras clinicas e liberação de resultados laboratoriais, além da sobrecarga de trabalho dos profissionais envolvidos nessa vigilância ${ }^{32}$, sobretudo em um cenário de pandemia, justificam as lacunas ora encontradas. Deve ser destacado a disponibilidade do banco de dados referentes a SRAG oportunamente, mesmo sujeito a alterações, permitindo a pesquisadores e profissionais de saúde o acesso a dados de interesse a saúde coletiva.

Este estudo apresentou o avanço da SRAG no território pernambucano no período que compreende o início da pandemia de COVID-19. Trata-se de um espalhamento desigual, com maior força em regiões distantes na Região Metropolitana. Conhecer os dados de SRAG de forma retrospectiva e prospectiva, bem como a utilização de técnicas de análise espacial são estratégias relevantes que auxiliam na compreensão do cenário da COVID-19 em sua forma grave e contribui para planejamento de ações de enfrentamento à pandemia em curto, médio e longo prazo em diferentes níveis de gestão. 


\section{Colaboradores}

APSC Silva e WV Souza contribuíram substancialmente para a concepção do manuscrito, análise e interpretação dos dados, revisão crítica do conteúdo. LTS Maia contribuiu significativamente na interpretação dos dados e revisão crítica do conteúdo; todos os autores aprovaram a versão final do manuscrito.

\section{Referências}

1. Ribeiro AF, Pellini ACG, Yu ALF, Kitagawa BY, Liphaus BL, Abdalla CSS. Influenza A (H1N1) no estado de São Paulo, emergência em saúde pública de importância internacional, resposta articulada, com ênfase na redução de morbimortalidade associada à pandemia. Bol Epidemiol Paul 2010; 7(84):12-17.

2. Bastos LS, Niquini RP, Lana RM, Villela DAM, Cruz OG, Coelho FC. COVID-19 e hospitalizações por SRAG no Brasil: uma comparação até a $12^{\mathrm{a}}$ semana epidemiológica de 2020. Cad Saúde Pública 2020; 36(4):e00070120.

3. Brasil. Ministério da Saúde (MS). Secretaria de Vigilância em Saúde. Boletim Epidemiológico. Influenza: Monitoramento até a Semana Epidemiológica 52 de 2019. Brasília: MS; 2019.

4. Werneck GL, Carvalho MS. A pandemia de COVID-19 no Brasil: crônica de uma crise sanitária anunciada. Cad Saúde Pública 2020; 36(5):e00068820.

5. Brasil. Ministério da Saúde (MS). Plataforma Integrada de Vigilância em Saúde. Notificação de casos pelo novo coronavírus (COVID-2019) [Internet]. [acessado 2020 Jun 20]. Disponível em: http://plataforma.saude.gov. br/novocoronavirus/dados-abertos/

6. Brasil. Ministério da Saúde (MS). Diretrizes para Diagnóstico e Tratamento da COVID-19 [Internet]. 2020 [acessado 2020 Jun 28]. Disponível em: https:// sbim.org.br/images/files/notas-tecnicas/ddtCOVID-19-200407.pdf.

7. Oliveira WK, Duarte E, França GVA, Garcia LP. How Brazil can hold back COVID-19. Epidemiol Serv Saúde 2020; 29(2):e2020044.

8. Croda JHR, Garcia LP. Resposta imediata da Vigilância em Saúde à epidemia da COVID-19. Epidemiol Serv Saúde 2020; 29(1):e2020002.

9. Moreira RS. COVID-19: unidades de terapia intensiva, ventiladores mecânicos e perfis latentes de mortalidade associados à letalidade no Brasil. Cad Saúde Pública 2020; 36(5):e00080020.

10. Pernambuco. Secretaria Estadual de Saúde. Plano Diretor de Regionalização [Internet]. [acessado 2020 Jun 28]. Disponível em: http://portal.saude.pe.gov.br/ sites/portal.saude.pe.gov.br/files/pdrconass-versao final1.doc_ao_conass_em_jan_2012.pdf

11. Brasil. Instituto Brasileiro de Geografia e Estatística (IBGE). Pernambuco. Panorama [Internet]. 2020 [acessado 2020 Jun 21]. Disponível em: https://cidades. ibge.gov.br/brasil/pe/panorama

12. Assunção RM, Barreto SM, Guerra HL, Sacuraí E. Mapas de taxas epidemiológicas: uma abordagem Bayesiana. Cad Saude Publica 1998; 14(4):713-723.

13. Brasil. Instituto Brasileiro de Geografia e Estatística (IBGE). Cartas e mapas [Internet]. 2014 [acessado 2020 Jun 20]. Disponível em: http://www.ibge.gov.br

14. Brasil. Atlas de Desenvolvimento Humano no Brasil [Internet]. 2013 [acessado em 2020 Jul 19]. Disponível em: http://atlasbrasil.org.br/2013/pt/consulta/

15. Centers for Disease Control and Prevention (CDC). Updated guidelines for evaluating public health surveillance systems: recommendations from the guidelines working group [Internet]. 2001 [acessado 2020 Jun 20]. Disponível em: https://stacks.cdc.gov/ view/cdc/13376 
16. Arabil YM, Murthy S, Webb S. COVID-19: a novel coronavirus and a novel challenge for critical care. Intensive Care Med 2020; 46:833-836.

17. Ji D, Zhang D, Xu J, Chen Z, Yang T, Zhao P, Chen G, Cheng G, Wang Y, Bi J, Tan L, Lau G, Qin E. Prediction for Progression Risk in Patients with COVID-19 Pneumonia: the CALL Score. Clin Infect Dis 2020; ciaa414.

18. Weiss $P$, Murdoch D. Clinical course and mortality risk of severe COVID-19. Lancet 2020; 395(10229):10141015.

19. Instituto para Redução de Riscos e Desastres de Pernambuco. Covid em tempo Real [Internet]. [acessado 2020 Jul 3]. Disponível em: https://www.irrd. org/COVID-19/

20. Pernambuco. Governo do Estado. Secretaria de Saúde. Secretaria Executiva de Vigilância em Saúde. Nota técnica No 6/2020. Assunto: Vigilância Epidemiológica e laboratorial na epidemia da COVID-19 [Internet]. 2020 [acessado 2020 Jul 3]. Disponível em: https://www. cievspe.com/novo-coronavirus-2019-ncov

21. Our World in data. Coronavirus (COVID-19) Testing Statistics and Research [Internet]. [acessado 2020 Jul 1]. Disponível em: https://ourworldindata.org/ coronavirus-testing

22. Pernambuco. Governo do Estado. Secretaria de Saúde. Secretaria Executiva de Vigilância em Saúde. Coronavírus COVID-19 - Casos Confirmados; $N^{o}$ 89/2020 [Internet]. 2020 [acessado 2020 Jul 1]. Disponível em: https://12ad4c92-89c7-4218-9e11-0ee 136fa4b92.filesusr.com/ugd/3293a8_f6a4c42646cd472 f852800541b183792.pdf

23. Brasil. Ministério da Saúde (MS). Secretaria de Vigilância em Saúde. Departamento de Vigilância das Doenças Transmissíveis. Guia para a Rede Laboratorial de Vigilância de Influenza no Brasil. Brasília: MS; 2016.

24. Montalvão EA. Avaliação de atributos do Sistema de Vigilância Sentinela da Síndrome Gripal no Município do Rio de Janeiro, Brasil, 2013-2014 [dissertação]. Rio de Janeiro: Fundação Oswaldo Cruz, Escola Nacional de Saúde Pública Sergio Arouca; 2017.

25. Pan American Health Organization (PAHO). Influenza Report EW 51-52/ Regional Update: Influenza \& Other Respiratory Viruses [Internet]. 2019 [acessado 2020 Jul 27]. Disponível em: https:// www.paho.org/hq/index.php?option $=$ com docman\&view=download\&category_slug=influenzaalso-includes-pandemic-h1n1-2009-2206\&alias $=51323$-regional-update-influenza-epidemiological -week-51-52-january-6-2020-1\&Itemid=270\&lang $=$ en
26. Public Health England. Surveillance of influenza and other respiratory viruses in the UK Winter 2018 to 2019 [Internet]. 2016 [acessado 2020 Jul 27]. Disponível em: https://assets.publishing.service.gov.uk/government/ uploads/system/uploads/attachment_data/file/839350/ Surveillance_of_influenza_and_other_respiratory_ viruses_in_the_UK_2018_to_2019-FINAL.pdf

27. Maciel JAC, Castro-Silva II, Farias MR. Análise inicial da correlação espacial entre a incidência de COVID-19 e o desenvolvimento humano nos municípios do estado do Ceará no Brasil. Rev Bras Epidemiol 2020; 23:e200057.

28. Monteiro CC, Dezanet LNC, França EB. Monitoramento de vírus respiratórios na região metropolitana de Belo Horizonte, 2011 a 2013. Epidemiol Serv Saúde 2016; 25(2):233-242.

29. Silva B. A interiorização da Covid-19 no Nordeste: temos infraestrutura de saúde? Conselho Regional de Economia (CORECON) [Internet]. 2020 [acessado 2020 Jul 24]. Disponível em: http://www.corecon-rn. org.br/2020/04/07/a-interiorizacao-da-covid-19-nonordeste-temos-infraestrutura-de-saude/

30. Andrade LA, Gomes DS, Góes MAO, Souza MSF, Teixeira DCP, Ribeiro CJN. Surveillance of the first cases of COVID-19 in Sergipe using a prospective spatiotemporal analysis: the spatial dispersion and its public health implications. Rev Soc Bras Med Trop 2020; 53:e20200287.

31. Pedrosa NL, Albuquerque NLS. Análise Espacial dos Casos de COVID-19 e leitos de terapia intensiva no estado do Ceará, Brasil. Cien Saude Colet 2020; 25(Supl. 1):2461-2468.

32. Lana RM, Coelho FC, Gomes MFC, Cruz OG, Bastos LS, Villela DAM. Emergência do novo coronavírus (SARS-CoV-2) e o papel de uma vigilância nacional em saúde oportuna e efetiva. Cad Saúde Pública 2020; 36(3):e00019620.

Artigo apresentado em 07/07/2020

Aprovado em 08/08/2020

Versão final apresentada em 10/08/2020 